\title{
Waveguide magnetoplasmonic structure based on ferrite garnet film
}

\author{
Georgy Basiladze, Aleksandr Dolgov, Vladimir Berzhansky, Andrey Karavainikov, and Anatoly Prokopov
}

V. I. Vernadsky Crimean Federal University, 295007 Simferopol, Russia

\begin{abstract}
The magnetoplasmonic structure, which is a planar multimode magnetooptical waveguide, $10 \mu \mathrm{m}$ thick with plasmon resonance $\mathrm{SiO}_{2} / \mathrm{Cu}$ coating on the surface is experimentally realized. With the total length of the magnetooptical waveguide $4.6 \mathrm{~mm}$ and the length of the $\mathrm{SiO}_{2} / \mathrm{Cu}$ coating equal to $3.9 \mathrm{~mm}$, the insertion loss of the structure for TM- and TE-polarized light was 22 and $4 \mathrm{~dB}$, respectively, at a wavelength of $1550 \mathrm{~nm}$. Linearly polarized light was launched into the magneto-optical waveguide using a SMF-28 single-mode optical fiber coupled to the polished input edge of the waveguide. The structure is of interest for use as miniature magnetically controlled modulators of light intensity.
\end{abstract}

\section{Introduction}

Planar waveguide structures based on dielectric magnetic films are of considerable interest due to the unique property of magneto-optical nonreciprocity and the ability to control the characteristics of the light propagating through them using an external magnetic field. The study of such structures is widely represented in the literature, where their properties are studied in detail and the possibility of using them as various planar optical devices, such as isolators [1], circulators [2], modulators [3], has been shown. At the same time, similar structures in which the actions of the Faraday effect in a dielectric magnetic waveguide and of surface plasmon resonance (SPR) in a metal layer on waveguide surface are combined are not sufficiently studied. This is especially true for structures based on sufficiently thick (8-10 $\mu \mathrm{m})$ dielectric magnetic waveguides, which can be relatively easily coupled to a telecomunication singlemode optical fiber. As follows from modern studies [4], the combination of magneto-optical and plasmon effects opens new prospects for the use of magnetic materials in sensor systems and miniature light-control devices. As an example of waveguide structures using dielectric magnetic materials and photon-plasmon interaction for controlling the parameters of an optical wave by means of an external magnetic field, devices theoretically developed in [5, 6] can serve. So, in [5] the possibility of creating Faraday rotator based on a hybrid ridgeplasmonic waveguide, including the yttrium iron garnet (Bi: YIG). In [6] the possibility of amplifying the nonreciprocity effect in a structure based on a silicon waveguide with a lattice plasmon structure on a surface covered with a dielectric magnetic material $\mathrm{Bi}$ : GIG is shown.

The aim of this work was the experimental creation a planar structure based on a waveguide from an liquid- phase epitaxial ferrite garnet film, about $10 \mu \mathrm{m}$ in thickness, with a plasmon resonance coating on the surface that is capable of absorbing a TM-polarized nearinfrared wavelength. As is well known [7-9] similar structures on the basis of not magnetic material well perform the function of the polarizer. In our case, when a magnetic material is used instead of a dielectric waveguide layer, the structure assumes the possibility of being used as an active element for various planar devices, for example, a light intensity modulator [10].

\section{Model of the structure}

It is well known [7-9] that the effect of the optical waveguide TE polarizer with a plasmon structure on the surface of the waveguide is based on the resonant coupling of the waveguide TM mode to the plasmon mode, which is supported by the metal layer due to the excitation of the surface plasmon resonance. Such a resonance arises under the condition of phase synchronization of the waveguide and plasmon TM modes. This condition is achieved due to the dielectric buffer layer, which is located between the dielectric waveguide and the metal layer. From the correctness of the choice of thicknesses and refractive indices of the metal and buffer layers for a given optical waveguide, the efficiency of quenching of the optical TM wave in the structure largely depends.

Fig. 1 shows the model of the waveguide magnetoplasmonic structure, the possibility of experimental realization and obtaining of a plasmon effect in which was verified in this work. Here are the substrate from the nonmagnetic gadolinium gallium garnet (GGG) $\mathrm{Gd}_{3} \mathrm{Ga}_{5} \mathrm{O}_{12}$ (1), the dielectric waveguide layer from the epitaxial film of ferrite garnet $(\mathrm{Bi}, \mathrm{Lu})_{3}(\mathrm{Fe}, \mathrm{Ga})_{5} \mathrm{O}_{12}$ (2), $\mathrm{SiO}_{2}$ buffer layer (3), metal layer (4). 


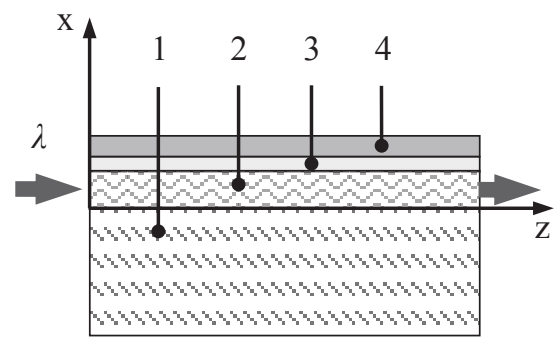

Fig. 1. Magnetoplasmonic structure: 1 - GGG substrate, 2 - ferrite garnet film, 3 - buffer layer 4 - metal layer.

\section{Fabricating the structure}

To realize the model, we used a dielectric magnetic film with a thickness $d_{\mathrm{f}}=10 \mu \mathrm{m}$ (refractive index $n_{\mathrm{f}} \approx 2.22$ at $\lambda=1550 \mathrm{~nm}$ ), which had a easy-plane magnetic anisotropy with relatively small external magnetic fields for its saturation along the hard $(\sim 4 \mathrm{Oe})$ and easy $(\sim 0,6$ Oe $)$ of the magnetization axes, with a specific Faraday rotation of $55.3^{\circ} / \mathrm{mm}$ at a wavelength of 1550 $\mathrm{nm}$. The film was grown by liquid-phase epitaxy from a melt solution on a substrate of GGG, which has a thickness of $d_{\mathrm{S}} \approx 500 \mu \mathrm{m}\left(n_{\mathrm{S}} \approx 1.92\right.$ at $\left.\lambda=1550 \mathrm{~nm}\right)$ end the orientations of the crystallographic axes (111)-plane. According to the AFM measurement, the root mean square roughness of the surface of the magnetic film is $1.682 \mathrm{~nm}$. The input and output edge of the magnetic film were polished along the hard magnetization axis so that light could be launched into film in the direction of the axis of easy magnetization. The distance between the polished ends was $L_{\mathrm{f}}=4.6 \mathrm{~mm}$, the size along the polished ends was $8 \mathrm{~mm}$. It should be noted that, in contrast to the plasmon structures presented in $[7,8]$, where the waveguide is single-mode, the magnetooptical waveguide used in this work, according to the above optical and geometric parameters, is a multimode.

We used easily accessible and relatively inexpensive materials such as fused quartz $\left(\mathrm{SiO}_{2}\right)$ and copper $(\mathrm{Cu}-$ $99.9 \%$ ) to deposit the buffer and metal layers on the surface of the magnetic film. Deposition was carried out by reactive ion-beam sputtering of the target in a vacuum chamber. Wherein, the possibilities of the equipment used to maintain the vacuum in the sputtering chamber were limited to a pressure of $1.6 \times 10^{-6} \mathrm{kPa}$. $\mathrm{SiO}_{2}$ and $\mathrm{Cu}$ were deposited on a sample of a magnetic film sequentially, each in its cycle - first a $\mathrm{SiO}_{2}$ buffer coating then a $\mathrm{Cu}$ layer.

$\mathrm{SiO}_{2}$ was deposited by sputtering a target from fused quartz in the presence of a gas mixture of argon and oxygen at a pressure of $9.6 \times 10^{-5} \mathrm{kPa}$, an accelerating voltage of $5 \mathrm{kV}$ and a current of $0.16 \mathrm{~A}$. The deposition rate was $8 \mathrm{~nm} / \mathrm{min}$. The gas mixture in the sputtering chamber, which was previously evacuated to a vacuum depth of $1.6 \times 10^{-6} \mathrm{kPa}$, was formed by the injection of argon to a pressure of $1.1 \times 10^{-5} \mathrm{kPa}$, then of oxygen to the working pressure in the chamber of $9.6 \times 10^{-5} \mathrm{kPa}$.

The buffer coating on the surface of the magnetic film was formed in the form of steps along the large side of the sample, each of which had its own thickness. This technique was used to increase the probability of achieving resonance in the fabricated structure. The stepped coating was formed with the aid of a special tooling having a movable shutter adjacent to the surface of the sample. This shutter, after the deposition of one of the $\mathrm{SiO}_{2}$ layers, moved a distance of $\sim 1 \mathrm{~mm}$ along the large side of the sample to deposit the next layer. As a result, a buffer coating was obtained from $\mathrm{SiO}_{2}$ with a thickness of steps $8,13,18$ and $23 \mathrm{~nm}$ varying along the large side of the sample. The dimensions of these steps along the large side of the sample were $1.2 \mathrm{~mm}, 1.3 \mathrm{~mm}$, $1.3 \mathrm{~mm}$ and $1 \mathrm{~mm}$, respectively, and $3.9 \mathrm{~mm}$ in the direction of light propagation.

$\mathrm{Cu}$ was deposited over the buffer coating, as well as on the adjacent non-coated portion of the magnetic film surface. Precipitation was carried out by sputtering a copper target in the presence of argon at a pressure of $7.7 \times 10^{-5} \mathrm{kPa}$ (residual gas partial pressure $1.6 \times 10^{-6}$ $\mathrm{kPa}$ ), accelerating voltage $3 \mathrm{kV}$ and current $75 \mathrm{~mA}$. The sample was located at a distance of $20 \mathrm{~cm}$ from the target. Wherein, the deposition rate of copper was $10 \mathrm{~nm} / \mathrm{min}$. The deposition time was $3 \mathrm{~min}$, the obtained copper layer thickness $d_{\mathrm{Cu}}=30 \mathrm{~nm}$. The size of the copper coating on the surface of the magnetic film along its large side was $6.2 \mathrm{~mm}$, and in the direction of light propagation $L_{\mathrm{Cu}}=3.9 \mathrm{~mm}$.

Fig. 2 shows a photograph of the surface of a structure with a dimensional partition into areas. Here are the area of the uncoated film (a), the area with a copper layer without buffer (b), $\mathrm{SiO}_{2} / \mathrm{Cu}$ heterostructures with $\mathrm{SiO}_{2}$ thicknesses, respectively, $8 \mathrm{~nm}$ (c), $13 \mathrm{~nm}$ (d), $18 \mathrm{~nm} \mathrm{(e)} \mathrm{and} 23 \mathrm{~nm}$ (f) at a thickness of $\mathrm{Cu}-d_{\mathrm{Cu}}=30 \mathrm{~nm}$.

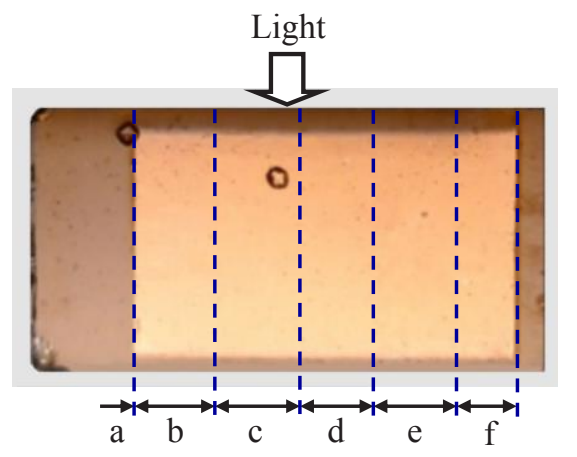

Fig. 2. The image of the surface of the structure (the size of the structure is $8 \times 4.6 \mathrm{~mm}$ ).

Thus, on the surface of the magneto-optical film for plasmon resonance studies, we had four plasmon $\mathrm{SiO}_{2} / \mathrm{Cu}$ heterostructures with different $\mathrm{SiO}_{2}$ thicknesses.

\section{Structure study}

Fig. 3 shows in a simplified form the design of the waveguide magnetoplasmon structure, realized in this work, and the scheme of the experiment for its investigation.

As a light source, a semiconductor laser with a wavelength of $1550 \mathrm{~nm}$ was used, to the output of which a fiber optic (FO) polarizer (not shown in the figure), made of a SMF-28 single-mode optical fiber was connected. Linearly polarized light from the FO 
polarizer is launched into the magnetic film 2 through its polished end-face connected to the fiber terminal 6 of the polarizer. Thus, by establishing the corresponding polarization direction at the input of the structure, it was possible to excite only the TE or only the TM mode in the magneto-optical waveguide. Wherein, to study different areas of the structure, the sample was moved in a horizontal plane by means of a precision positioner.

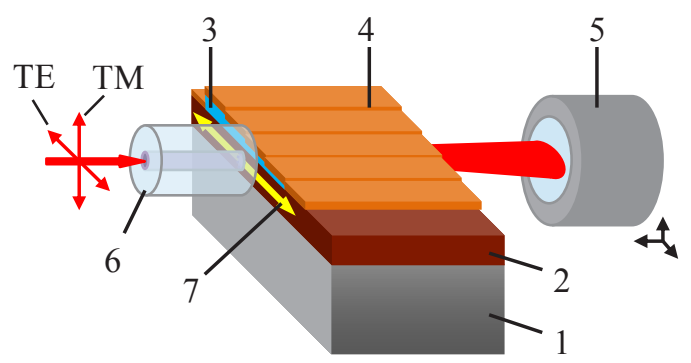

Fig. 3. Scheme of the experiment: 1-GGG substrate $\left(d_{\mathrm{s}} \approx 500 \mu \mathrm{m}\right), 2-$ ferrite garnet film $\left(d_{\mathrm{f}}=10 \mu \mathrm{m}\right), 3-\mathrm{SiO}_{2}$ buffer layer with a step profile $\left(d_{\mathrm{SiO} 2}=8-23 \mathrm{~nm}\right), 4-\mathrm{Cu}$ layer $\left(d_{\mathrm{Cu}}=30 \mathrm{~nm}\right), 5-\mathrm{Ge}$ photodiode, 6 - optic fiber, 7 -sample scanning direction with respect to the fiber.

Measurements of the signals at the output of the structure were performed using a germanium photodiode 5 and a photocurrent detector (not shown in Fig. 3). Wherein, the light attenuation in the structure was calculated for each of the polarizations at the input by the formula:

$$
\alpha=-10 \times \log \left(I_{0} / I_{\text {out }}\right)
$$

where $I_{0}$ and $I_{\text {out }}$ are the light intensities at the input and output of the magnetic film. When performing measurements, the film was magnetized by an external magnetic field of strength $>50 \mathrm{Oe}$ in the plane, transverse to the direction of light. For this purpose, permanent magnets located on the sides of the sample (not shown in Fig. 3) were used.

\section{Results and discussion}

Fig. 4 shows the optical loss of TM- and TE-polarized light beams in the magnetoplasmonic structure as a function of the coordinate of a launch into the input edge of the waveguide. The coordinate of the launch of the light beam into the waveguide was changed by scanning the sample in a horizontal plane relative to the fiber. Vertical dashed lines show the boundaries between areas of the surface with different coating compositions (See Fig. 2). The noise level in these dependencies does not exceed $\pm 0.9 \mathrm{~dB}$.

It can be seen from Fig. 4 the attenuation of light for both polarizations during propagation in a magnetic film along a-area, where any coating is absent does not exceed $4 \mathrm{~dB}$. Then, in the transition to b-area, where the copper layer does not have a buffer, for TM-polarized light, the attenuation increases to a level of $7.5 \mathrm{~dB}$ and continues to increase in the $\mathrm{c}\left(d_{\mathrm{SiO} 2}=8 \mathrm{~nm}\right)$ and $\mathrm{d}$ $\left(d_{\mathrm{SiO} 2}=13 \mathrm{~nm}\right)$ regions, reaching in the d-area of the largest value $\approx 22 \mathrm{~dB}$. With a further thickening of the buffer layer in e $\left(d_{\mathrm{SiO} 2}=18 \mathrm{~nm}\right)$ and $\mathrm{f}\left(d_{\mathrm{SiO} 2}=23 \mathrm{~nm}\right)$ regions, a certain decrease in the curve for the TM polarization is observed. Simultaneously, for TEpolarized light, the attenuation level within the experimental accuracy can be considered independent of the thickness of the buffer layer. According to the figure, this attenuation level on the average corresponds to a value of about $4 \mathrm{~dB}$.

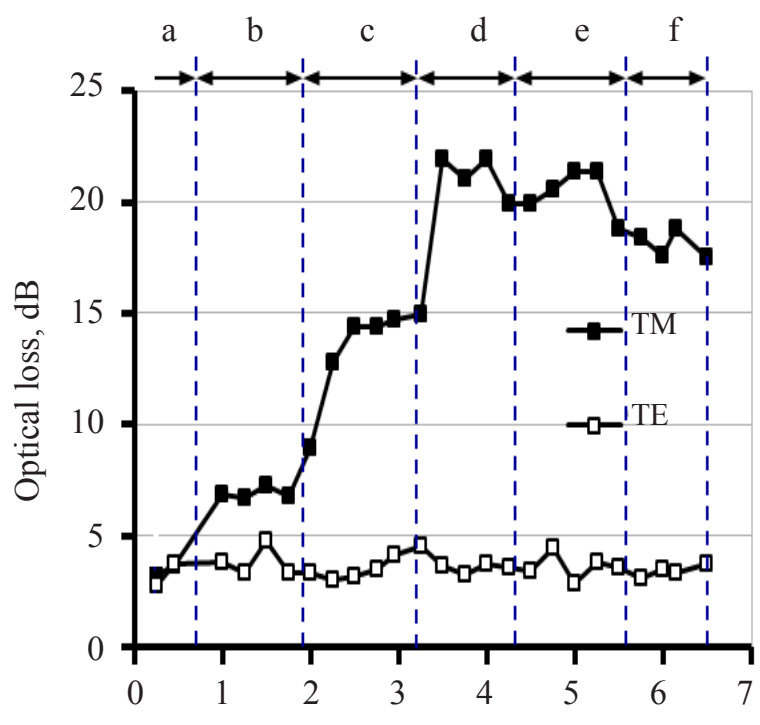

Distance along the waveguide edge, $\mathrm{mm}$

Fig. 4. TM, TE mode loss vs. $\mathrm{SiO}_{2}$ layer thickness, where $d_{\mathrm{SiO} 2}$ : $0 \mathrm{~nm}(\mathrm{a}, \mathrm{b}), 8 \mathrm{~nm}$ (c), $13 \mathrm{~nm}$ (d), $18 \mathrm{~nm}$ (e), $23 \mathrm{~nm}(\mathrm{f}) ; d_{\mathrm{cu}}$ : $0 \mathrm{~nm}$ (a), $30 \mathrm{~nm}$ (b-f).

The explanation of the physical mechanism of damping of TM-polarized light in the structure, in our opinion, logically follows from the behavior of the obtained dependence on the thickness of the buffer layer (see Fig. 4). In this regard, one should note a certain similarity of the above dependence with the theoretically obtained [11, 12] and experimentally [8] curves for structures in which the resonance interaction of the waveguide and plasmon-polariton waves is the dominant damping mechanism. The similarity is that, as in our case, with a thickening of the buffer layer to a thickness where the resonance is maximal, the damping of the TM mode increases. If there was no such mechanism in our structure, then at an increase in the thickness of the buffer layer, an increase in the optical signal would be observed, since the dominant mechanism of ohmic losses would undergo a decrease due to a decrease in the fraction of the light energy penetrating into the metal.

Thus, we come to the conclusion that the nature of the attenuation of TM-polarized light in the resulting structure is directly related to the resonance interaction of the waveguide and plasmon-polariton waves. Such interaction is first amplified by equalizing the phase velocities of these waves with an increase in the thickness of the buffer layer from 0 to $13 \mathrm{~nm}$. Then, with further thickening of the buffer layer and caused by this attenuation of the interaction between the waveguide and plasmon-polariton waves, the attenuation of the TM polarized waveguide mode decreases. The comparatively 
small efficiency of the observed resonance can be explained by the insufficient optimization of the parameters of the buffer and metal layers, as well as by the multimode nature of the optical waveguide system. The latter factor can lead to a significant transformation of the transverse distribution of the light wave field as it propagates in the waveguide. Therefore, initially the high degree of localization of the field at the metal interface, which is inherent in the lowest order TM mode in the metallized waveguide [12, 13], can be gradually broken, resulting in a disruption of the connection with the plasmon polariton.

With regard to the possibility of modulating the intensity of light by means of the proposed structure, it can be realized by the action of an external alternating magnetic field directed parallel to the light propagation path. The Faraday rotation of the plane of light polarization caused by such an action will lead to an angular vibration of the direction of light polarization in the magnetic film. At those times when the direction of polarization is perpendicular to the plane of the film, the output of the device will exhibit a minimum of light intensity caused by the transformation of the energy of the waveguide TM mode into a plasmon mode. When the light is polarized in the plane of the magnetic film, the intensity will be maximum, since the TE wave propagates without exciting plasmon oscillations in the structure. Thus, be efficiency of transforming the TMpolarized component of the electromagnetic field of the waveguide mode into plasmon mode will determine such an important modulator characteristic as the modulation depth. Based on the maximum difference between the attenuation values for TM- and TE-polarized signals $(\approx 22 \mathrm{~dB}$ and $\approx 4 \mathrm{~dB}$, respectively), this parameter for a modulator based on the sample considered in this paper can be about $96 \%$.

A certain feature of the technology used to make the investigated sample of the magnetoplasmonic structure is a relatively low level of vacuum and a relatively low purity of the sputtered materials that form a plasmon heterostructure on the surface of the magnetic film. At the same time, in accordance with the current recommendations [14], to obtain high-quality plasmon coatings, it is necessary to adhere to the more stringent requirements for the purity of plasmon materials $(\geq 99.99 \%)$ and the pressure in the vacuum chamber of $<10^{-5}$ Torr $\left(\approx 1.333 \times 10^{-6} \mathrm{kPa}\right)$. It is logical to assume that the fulfillment of these conditions can lead to a significant increase of the efficiency of plasmon resonance in the considered structure.

\section{Conclusion}

The possibility of fabricating a waveguide the magnetoplasmonic structure on the basis of a planar multimode waveguide, which is an epitaxial film of a garnet ferrite with a $\mathrm{SiO}_{2} / \mathrm{Cu}$ resonant heterostructure on the surface, is demonstrated experimentally. A feature of the technology of deposition of a resonant heterostructure using the method of reactive ion beam sputtering of the target is a relatively high baseline pressure in the vacuum chamber $\left(>10^{-5}\right.$ torr) and a relatively low purity of the initial metal for sputtering $(\sim 99.9 \%)$. The thus obtained plasmon resonance $\mathrm{SiO}_{2} / \mathrm{Cu}$ coating $\left(d_{\mathrm{SiO} 2}=13 \mathrm{~nm}, \quad d_{\mathrm{Cu}}=30 \mathrm{~nm}\right), L_{\mathrm{Cu}}$ $=3.9 \mathrm{~mm}$ in length with a total waveguide length of $L_{\mathrm{f}}$ $=4.6 \mathrm{~mm}$ and its thickness of $d_{\mathrm{f}}=10 \mu \mathrm{m}$, provided quenching of the introduced TM-polarized wave $(\lambda=1550 \mathrm{~nm})$ about $22 \mathrm{~dB}$ and insertion loss for the TEpolarized wave about $4 \mathrm{~dB}$. The efficiency of the resonance coupling of the optical and plasmon modes in such a structure can be substantially increased by optimizing the thickness of the buffer and metallic layers, as well as the technology of their deposition.

The waveguide magnetoplasmonic structure presented in this paper can be used as an active element of a miniature magnetooptical light intensity modulator in planar design [10].

\section{Acknowledgement}

This work is support by the RF Ministry of Education and Science in the framework of the state task (project no. 3.7126.2017/8.9).

\section{References}

1. J.P. Castera, G. Hapner, Appl. Opt. 16, 8, 20312034, (1977)

2. R. Wolfe, R.A. Lieberman, V.J. Fratello, R.E. Scotti, N. Kopylov, Appl. Phys. Lett. 56, 5, 426-428 (1990)

3. S.E. Irvine, A.Y. Elezzabi, Opt. Commun, 220, 325329 (2003)

4. G. Armelles, A. Cebollada, A. García-Martín, M.U. González, Adv. Opt. Mater. 1, 10-35 (2013)

5. C. J. Firby, PoHan Chang, Amr S. Helmy, and A. Y. Elezzabi. ACS Phot. 3, 2344 (2016)

6. L. Halagacka, K. Postava, M. Vanwolleghem, B. Dagensb, and J. Pi`stora. Proc. of SPIE Vol. 9516, 95161 (2015)

7. M.N. Zervas, I.P. Giles, Opt. Lett. 15, 9, 513-515 (1990)

8. I.V. Il'ichev, N.V. Toguzov, A.V. Shamray, Tech. Phys. Lett. 35, 9, 831-833 (2009)

9. A. Kumar, Rakesh, M. Kumar, G. Bhatt, A. Kapoor, Intern. J. Eng. \& Appl. Sciences, 7, 2, 31-36 (2015)

10. G.D. Basiladze, V.N. Berzhansky, A.I. Dolgov, Patent RU 161388 (2016)

11. J.N. Polky, G.L. Mitchell, J. Opt. Soc. Am. 64, 3, 274-279 (1974)

12. Y. Yamamoto, T. Kamiya, H. Yanai, IEEE J. Quant. Electron. QE-11, 9, 729-736 (1975)

13. S.C. Rashleigh, Opt. \& Quant. Electronics 8, 49-60 (1976)

14. K.M. McPeak, S.V. Jayanti, S.J.P. Kress, S. Meyer, S. Iotti, A. Rossinelli, D.J. Norris, ACS Photonics, 2, 326-333 (2015) 